Article

\title{
Dietary Supplementation with Dunaliella Tertiolecta Prevents Whitening of Brown Fat and Controls Diet-Induced Obesity at Thermoneutrality in Mice
}

\author{
Yukari Yamashita ${ }^{1}$, Tamaki Takeuchi ${ }^{1}$, Yuki Endo ${ }^{1}$, Ayumi Goto ${ }^{1}{ }^{\circledR}$, Setsuko Sakaki ${ }^{2}$, \\ Yuji Yamaguchi ${ }^{2}$, Hiroyuki Takenaka ${ }^{2}$ and Hitoshi Yamashita ${ }^{1, *}$ \\ 1 Department of Biomedical Sciences, College of Life and Health Sciences, Chubu University, Kasugai \\ 487-8501, Japan; yappy8008@na.commufa.jp (Y.Y.); takeuchi@isc.chubu.ac.jp (T.T.); \\ yendo@isc.chubu.ac.jp (Y.E.); a-goto@isc.chubu.ac.jp (A.G.) \\ 2 MAC Gifu Research Institute, MicroAlgae Corporation, 4-15 Akebono, Gifu 500-8148, Japan; \\ sakaki@mac-bio.co.jp (S.S.); yamaguchi@mac-bio.co.jp (Y.Y.); takenaka@mac-bio.co.jp (H.T.) \\ * Correspondence: hyamashi@isc.chubu.ac.jp; Tel./Fax: +81-568-51-6017
}

Received: 14 May 2020; Accepted: 2 June 2020; Published: 5 June 2020

\begin{abstract}
We investigated the effect of evodiamine-containing microalga Dunaliella tertiolecta (DT) on the prevention of diet-induced obesity in a thermoneutral C57BL/6J male $\left(30^{\circ} \mathrm{C}\right)$. It attenuates the activity of brown adipose tissue (BAT), which accelerates diet-induced obesity. Nine-week-old mice were fed a high-fat diet supplemented with $10 \mathrm{~g}$ (Low group) or $25 \mathrm{~g}$ (High group) DT powder per $\mathrm{kg}$ food for 12 weeks. Compared to control mice without DT supplementation, body weight gain was significantly reduced in the High group with no difference in food intake. Tissue analyses indicated maintenance of multilocular morphology in BAT and reduced fat deposition in liver in DT-supplemented mice. Molecular analysis showed a significant decrease in mammalian target of rapamycin-ribosomal S6 protein kinase signaling pathway in white adipose tissue and upregulation in mRNA expression of brown fat-associated genes including fibroblast growth factor-21 ( $F g f 21)$ and uncoupling protein $1(U c p 1)$ in BAT in the High group compared to the control. In the experiments using C3H10T1/2 adipocytes, DT extract upregulated mRNA expression of brown fat-associated genes in dose-dependent and time-dependent manners, accompanied by a significant increase in secreted FGF21 levels. Our data show the ability of DT as a nutraceutical to prevent brown fat attenuation and diet-induced obesity in vivo.
\end{abstract}

Keywords: brown fat; fibroblast growth factor-21; microalga; obesity; thermoneutrality; uncoupling protein 1

\section{Introduction}

Adipose tissues are largely responsible for lipid and glucose metabolism, affecting energy homeostasis under the control of various hormones and cytokines in the body. Obesity is characterized by excess fat deposition mainly in white adipose tissue (WAT) and subsequently leads to fat accumulation in the liver, which is a serious health risk in industrialized societies [1]. In contrast to WAT, brown adipose tissue (BAT) can undergo thermogenesis mediated by mitochondrial uncoupling protein 1 (UCP1), which maintains homeothermy in mammals in a cold environment $[2,3]$. As UCP1-mediated thermogenesis dissipates caloric energy as heat, BAT also plays a crucial role in energy metabolism. UCP1 deficiency increases susceptibility to diet-induced obesity (DIO) with age in mice [4]. Adult humans have functional BAT, but it was found to decrease with age and showed an inverse correlation with the degree of adiposity [5]. This underscores the importance of considering BAT as a potential tissue in the treatment of obesity and metabolic syndrome [6,7]. Moreover, discovery 
of endocrine factors such as fibroblast growth factor 21 (FGF21), which induces UCP1-expressing brown-like adipocytes (beige adipocytes) in WAT, have accelerated basic and clinical studies on the stimulators of brown/beige fat formation and activity $[7,8]$.

Microalgae are good sources of various nutraceuticals such as vitamins, carotenoids, polyunsaturated fatty acids, sterols, and proteins [9]. These constituents from Spirulina, Chlorella, Dunaliella, Scenedesmus, and Phaeodactylum have been reported to have various health benefits including anti-oxidant, anti-inflammatory, and anticancer properties. They also help reduce blood pressure, cholesterol, and body weight $[9,10]$. Kumar et al. reported that supplementation of Scenedesmus dimorphus and Schroederiella apiculata in rat attenuates obesity and its associated pathology such as glucose intolerance and fatty liver [11]. Gille et al. have recently demonstrated that daily oral injection of ethanolic extract of Phaeodactylum tricornutum improved diet-induced obesity in mice at $22{ }^{\circ} \mathrm{C}$ [12]. This study demonstrated an upregulation of Ucp $1 \mathrm{mRNA}$ in inguinal WAT (IWAT) and an increase in its protein level in interscapular BAT (IBAT) in mice administered with the microalga extract compared to those in control mice, which could be caused by the effect of fucoxanthin.

Evodiamine (Evo) is a major alkaloid compound extracted from the fruit of Evodia fructus (Evodia rutaecarpa Benth., Rutaceae), which has been used as a traditional Chinese herbal medicine for the treatment of pain, vomiting, and pyresis. Numerous studies have demonstrated that Evo exhibits anti-nociceptive, anti-obesity, anti-tumor, anti-inflammatory, and vasodilatory effects [13-15]. We have also demonstrated that it prevents body weight gain in diet-induced, age-related, and genetic obesity in mice [16-18]. In these studies, we found that Evo inhibits insulin-stimulated mammalian target of rapamycin (mTOR)-ribosomal S6 protein kinase (S6K) activation in 3T3-L1 adipocytes and WAT, a mechanism which contributes to the improvement of obesity and insulin resistance in obese models. Thus, Evo shows a great potential for the treatment of metabolic diseases including obesity and diabetes.

Recently, we discovered several species of microalgae containing evodiamine, of which Dunaliella tertiolecta (DT) produced the highest level of Evo [19]. DT is a strictly photoautotrophic, unicellular chlorophyte with a single cup-shaped chloroplast and a photosynthetic apparatus similar to that of higher plants [20]. DT has been reported to exhibit skeletal muscle relaxant activity [21], plasma cholesterol-reducing activity [22], and antiaggregant activity [23]. However, whether this microalga exhibits anti-obesity effect has not yet been investigated. As DT contains Evo, this green microalga may prevent obesity. Therefore, in the present study, we aimed to clarify the potential of this microalga as a food supplement for prevention of obesity in mice. In addition, we performed animal experiments under thermoneutral condition at $30^{\circ} \mathrm{C}$, in which BAT thermogenesis including UCP1 expression is greatly attenuated, increasing susceptibility to diet-induced obesity, and thereby leading us to strictly assess the effect of DT on brown fat formation and obesity. This is the first report showing the effects of DT on FGF21 production, brown fat formation, and inhibition of diet-induced obesity.

\section{Materials and Methods}

\subsection{Microalgae Cultivation and Preparation of Algal Extract}

DT and Dunaliella salina (DS) were grown in outdoor open raceway ponds at the MAC Miyako farm in Japan as previously described [19]. Sample preparation and evodiamine quantification were performed according to our protocol [19]. In the preparation of DT and DS extracts, $6 \mathrm{~g}$ of dried biomasses were extracted with $100 \mathrm{~mL}$ ethanol in a reflux device at $90^{\circ} \mathrm{C}$ for $1 \mathrm{~h}$. After the extracts were centrifuged at $3200 \mathrm{rpm}$ for $5 \mathrm{~min}$, the supernatants were filtered using filter papers. The filtrates were then concentrated to a final volume of $50 \mathrm{~mL}$ by a rotary evaporator apparatus at $90^{\circ} \mathrm{C}$. The Evo content in the extract was estimated to be 0.0474 and $0.007 \mu \mathrm{g} / \mathrm{mL}$ in DT and DS, respectively. 


\subsection{Experimental Animals}

Inbred C57BL/6J mice were maintained under artificial lighting for $12 \mathrm{~h}$ per day and provided with standard chow (11.6\% fat; Diet No. CE-2, CLEA Japan, Inc., Shizuoka, Japan) and tap water ad libitum in our animal facility at $30^{\circ} \mathrm{C}$. Nine-week-old male C57BL/6J mice were divided randomly into three groups and were fed a high-fat diet (HFD) (41.9\% fat; Diet No. D15040, CLEA Japan, Inc.) with $0 \mathrm{~g}, 10 \mathrm{~g}$, or $25 \mathrm{~g}$ DT powder per $\mathrm{kg}$ food (corresponding to 0, 4 and $10 \mu \mathrm{g}$ Evo per $\mathrm{kg}$ food, respectively) for 12 weeks (Figure 1). These mice were housed individually. Body weight and food intake were measured once per week. Tissue and blood samples were collected and stored at $-80{ }^{\circ} \mathrm{C}$ or were fixed in $4 \%$ paraformaldehyde dissolved in phosphate-buffered saline (PBS). All animal experiments were performed in strict accordance with the recommendations of the Fundamental Guidelines for Proper Conduct of Animal Experiments and Related Activities in Academic Research Institutions under the jurisdiction of the Ministry of Education, Culture, Sports, Science, and Technology, Japan. The protocol was approved by the Institutional Animal Care and Use Committee of Chubu University (\#2810013).

Thermoneutral room $\left(\right.$ at $\left.30^{\circ} \mathrm{C}\right)$

\begin{tabular}{|c|c|c|}
\hline $\begin{array}{c}\text { Standard } \\
\text { chow }\end{array}$ & High-fat diet (HFD) \\
\hline $\begin{array}{c}\text { C57BL/6J } \\
\text { male }\end{array}$ & $\begin{array}{c}\text { Low group }(+10 \mathrm{~g} \text { DT powder per kg food) } \\
\text { High group }(+25 \mathrm{~g} \text { DT powder per kg food) }\end{array}$ & Analysis \\
\hline
\end{tabular}

Figure 1. Protocol of animal experiment. Nine-week-old mice were fed the high-fat diet (HFD) supplemented with or without Dunaliella tertiolecta (DT) powder for 12 weeks.

\subsection{Blood Biochemistry}

Blood samples were collected from the tail vein and immediately used to determine glucose level using a glucometer (NovoAssist Plus, Novo Nordisk, Tokyo, Japan). Serum insulin level was measured using enzyme-linked immunosorbent assay kits (Lebis-insulin-mouse, Sibayagi, Gunma, Japan). Serum triglyceride (TG) and total cholesterol (TC) levels were measured using TG E-test and T-Cho E-test kits (Wako Pure Chemicals, Osaka, Japan), respectively. Total liver lipids were extracted using the previously described protocol with minor modifications [24]. Briefly, liver samples (100 mg) were homogenized in $1 \mathrm{~mL}$ of hexane/isopropyl alcohol $(3: 2, v / v)$. The homogenized samples were centrifuged, and the supernatant was evaporated under reduced pressure. The dried samples were then resuspended in 10\% triton X-100 prepared in isopropyl alcohol, and the lipid levels were assayed using commercial kits. FGF21 levels in serum and culture medium were measured using mouse FGF-21 DuoSet ELISA (R\&D SYSTEMS, Minneapolis, MN, USA).

\subsection{C3H10T1/2 Adipocyte Culture}

C3H10T1/2-clone 8 cells (\#IF050415) were obtained from Health Science Research Resources Bank (Osaka, Japan). Cells were inoculated into multi-well plates and grown in Dulbecco's Modified Eagle Medium (DMEM, Wako Pure Chemicals) containing $10 \%$ calf serum (Biowest, Nuaillé, France) at $37^{\circ} \mathrm{C}$ in $5 \% \mathrm{CO}_{2}$. Once confluent, the cells were differentiated by maintaining them in DMEM supplemented with $10 \%$ fetal bovine serum (FBS, ICN, Irvine, CA, USA), $10 \mu \mathrm{g} / \mathrm{mL}$ insulin, $1 \mu \mathrm{M}$ dexamethasone, $0.5 \mathrm{mM}$ 3-isobutyl-1-methylxanthine, $125 \mu \mathrm{M}$ indomethacin, and $3 \mathrm{nM}$ T3 for 2 days, and then in 10\% FBS/DMEM containing insulin and T3 for 5 days. On day-7 after differentiation, the cells were washed with serum-free DMEM and treated with DT extract or DS extract in the absence of above stimulators and serum for different time periods indicated in time course experiments and $18 \mathrm{~h}$ for all the other experiments. 


\subsection{Protein Analysis}

Western blot analyses were performed on C3H10T1/2 cell lysates and total tissue lysates of epididymal WAT (EWAT), IBAT, and liver as described previously [16]. The concentrations of protein in the lysates were measured using BCA protein assay (Pierce Biotechnology, Rockford, IL, USA). Proteins were separated on 4-20\% SDS-polyacrylamide gels (Daiichi Pure Chemicals, Tokyo, Japan) and transferred onto Immobilon polyvinylidene difluoride membranes (Millipore, Bedford, MA, USA). The membranes were incubated with specific antibodies against mTOR, phospho-Ser2448 mTOR, p70S6 kinase, phospho-Thr389 p70S6 kinase, ribosomal protein S6 (rpS6), phospho-Ser235/236 rpS6, $\alpha / \beta$-tubulin (all from Cell Signaling Technology, Danvers, MA, USA), or UCP1 (ab10983, Abcam, Cambridge, UK). After incubation with the appropriate secondary antibody for $1 \mathrm{~h}$ at room temperature, specific signals were detected using the Immobilon western chemiluminescent HRP substrate (Millipore, Billerica, MA, USA). The resulting images were quantified using NIH Image (version 1.63).

\subsection{Gene Expression Analysis}

Total RNA was extracted using TRI REAGENT (Molecular Research Center, Inc., Cincinnati, OH, USA), according to the manufacturer's protocol. To quantify mRNA expression levels, RNAs from tissues or cultured cells were reverse-transcribed using High Capacity cDNA Reverse Transcription kits (Applied Biosystems, Foster City, CA, USA), as per manufacturer's instructions, and real-time RT-PCR analysis was performed using a Light-Cycler and THUNDERBIRD SYBR qPCR Mix (TOYOBO, Osaka, Japan). All gene expression data were normalized relative to 36B4 levels. The oligonucleotide primer sequences are mentioned in Table 1.

Table 1. Real-time RT-PCR primer sequences for mouse genes.

\begin{tabular}{ccc}
\hline Gene Symbol & Sense & Antisense \\
\hline $36 b 4$ & TCATCCAGCAGGTGTTTGACA & CCCATTGATGATGGAGTGTGG \\
Cidea & ATCACAACTGGCCTGGTTACG & TACTACCCGGTGTCCATTTCT \\
$F g f 21$ & GTGTCAAAGCCTCTAGGTTTCTT & GGTACACATTGTAACCGTCCTC \\
$P p a r g c 1 a$ & TAGGCCCAGGTACGACAGC & GCTCTTTGCGGTATTCATCC \\
$P r d m 16$ & GACATTCCAATCCCACCAGA & CACCTCTGTATCCGTCAGCA \\
$U c p 1$ & GTGAAGGTCAGAATGCAAGC & AGGGCCCCCTTCATGAGGTC \\
\hline
\end{tabular}

\subsection{Histological Analysis}

Tissue samples were removed and immediately fixed by immersion in $10 \%$ formaldehyde prepared in neutral buffer solution (Kishida Chemical, Osaka, Japan) at $4{ }^{\circ} \mathrm{C}$. They were then dehydrated, cleared, and paraffin-embedded. Paraffin sections $(4 \mu \mathrm{m})$ were stained with hematoxylin and eosin.

\subsection{Statistical Analysis}

Data are expressed as mean \pm standard error of the mean (SE). Statistical analyses were performed using the StatView (SAS Institute) programs. Significance in differences among more than two groups (from three to six in our study) were assessed using one-way analysis of variance (ANOVA). Two-Way Repeated Measures ANOVA followed by protected Fisher's Least Significant Difference post-hoc test were applied to determine statistical differences in body weight gain and food intake. Values of $p<0.05$ were considered statistically significant.

\section{Results}

\subsection{Effect of Evodiamine-Containing DT Supplementation on Diet-Induced Obesity at Thermoneutrality in Mice}

In comparison to the control group, body weight gain was significantly lower in the High group, but not in the Low group, under HFD and thermoneutral temperature conditions (Figure 2A), although 
there was no difference in the amount of food consumed among the three groups (Figure 2B). IBAT weight was significantly low and a reduction in EWAT weight was observed in the Low and High groups ( $p=0.0811$ and 0.0558 , respectively) compared to those in the control groups (Figure 2C). As expected, histological analysis showed a morphological change in IBAT in the control group at thermoneutrality, where the typical multilocular brown adipocyte morphology changed to white adipocyte-like unilocular morphology, a phenomenon called BAT whitening (Figure 2D). Intriguingly, the multilocular brown adipocyte morphology was maintained in IBAT in case of DT-supplemented mice, especially in the High group, compared to that of the control mice at $30^{\circ} \mathrm{C}$ (Figure 2D). In addition, a decrease in adipocyte size was observed in IWAT and EWAT of DT-treated groups compared to that of the control group (Figure 2D). There was no significant difference in serum glucose levels among the three groups (Figure 2E). However, insulin levels tended to decrease in DT-treated groups relative to control (Figure 2F). There were no significant differences in triglyceride (TG) and total cholesterol (TC) among the three groups (Figure 2G), whereas hepatic TG and TC levels were significantly lower in Low and/or High group than the control group (Figure $2 \mathrm{H}, \mathrm{I}$ ), indicating reduced fat deposition in liver in DT-treated mice. These results suggested that DT supplementation improved diet-induced obesity and associated fatty liver in vivo.
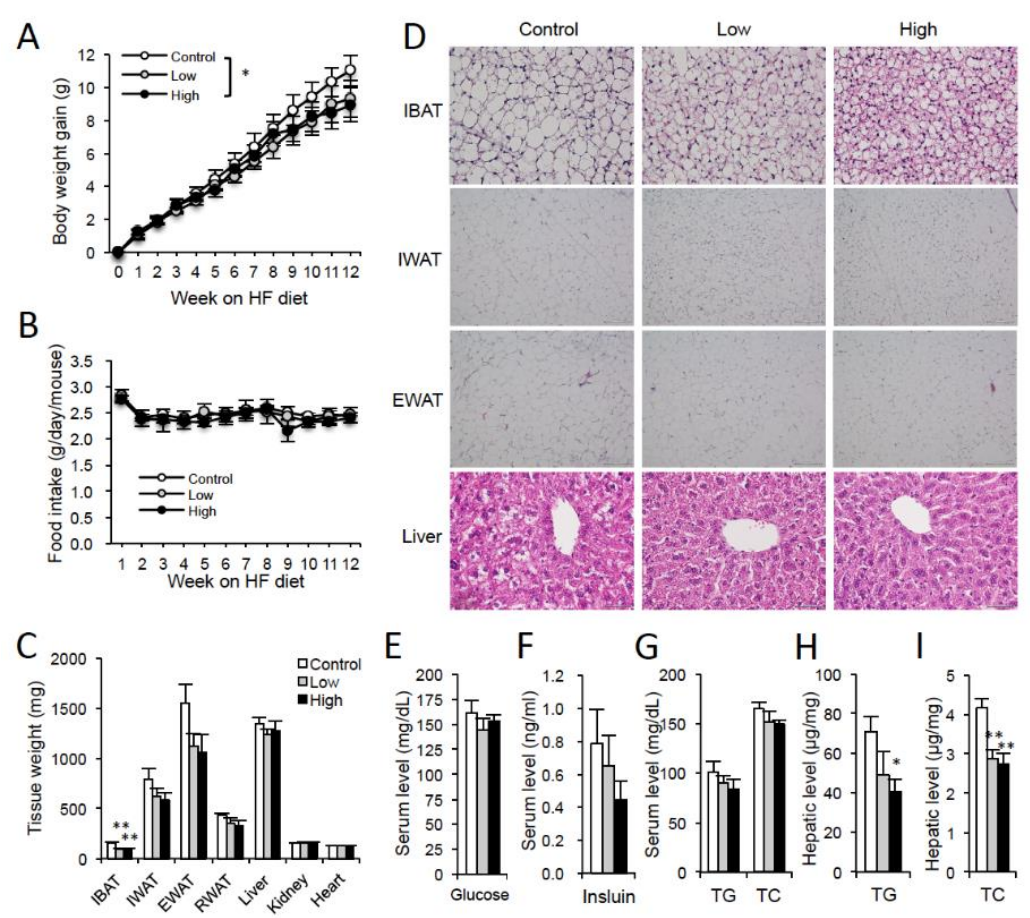

Figure 2. Effect of DT supplementation on diet-induced obesity at thermoneutrality in mice. Nine-week-old male C57BL/6J mice were fed the HFD supplemented with $0 \mathrm{~g}$ (Control), $10 \mathrm{~g}$ (Low) or $25 \mathrm{~g}$ (High) DT powder per kg food for 12 weeks. (A) Body weight gain. (B) Food intake. (C) Tissue weights. IBAT: interscapular BAT, IWAT: inguinal WAT, EWAT: epidydimal WAT, RWAT: retroperitoneal WAT. (D) Histology of IBAT, IWAT, EWAT, and liver were analyzed. Tissue sections were stained with hematoxylin and eosin. Representative images are shown. Scale bars, $50 \mu \mathrm{m}$ for IBAT and liver, $200 \mu \mathrm{m}$ for IWAT and EWAT. (E-G) Serum levels of fed glucose (E), insulin (F), triglyceride: TG, and total cholesterol: TC $(\mathbf{G})$ were examined. $(\mathbf{H}, \mathbf{I})$ Hepatic levels of TG (H) and TC (I) were also analyzed. Data are expressed as the mean \pm SE (Control: $n=5$, Low: $n=6$, High: $n=5) .{ }^{*} p<0.05,{ }^{* *} p<0.01$ vs. Control.

\subsection{Effect of DT Supplementation on Signal Transduction in WAT and Liver}

We also examined the effect of DT supplementation on mTOR signaling pathway in EWAT and liver in mice, as DT contains Evo, which is known to inhibit mTOR-S6K signaling in WAT in obese/diabetic mice [17]. As expected, the phosphorylation of mTOR Ser2448 and S6K Thr389 in EWAT 
significantly decreased in the High group compared to those in the control group and the Low group (Figure 3A,B). In addition, the phosphorylation of rpS6 Ser235/236, which is phosphorylated by S6K, was significantly reduced in EWAT in the High group compared to that in the control group (Figure 3C). On the other hand, there were no differences in the phosphorylation levels of mTOR and S6K in liver among the three groups (Figure 3D,E), whereas a significantly lower phosphorylation level of rpS6 was detected in the High group than the control group (Figure 3F). These results indicated that dietary supplementation of DT with a percentage content of $2.5 \%$ downregulated mTOR-S6K signaling in EWAT of mice with diet-induced obesity at thermoneutrality.

A

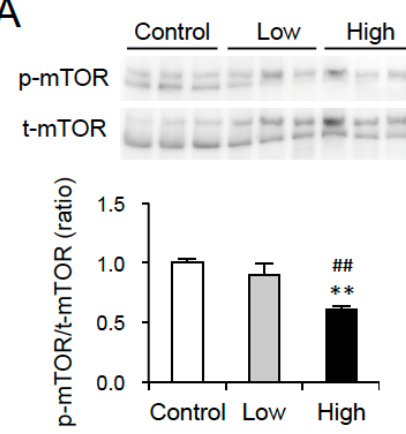

B
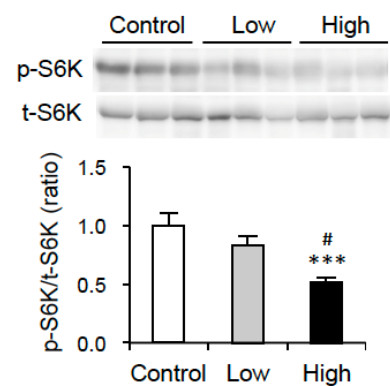

C
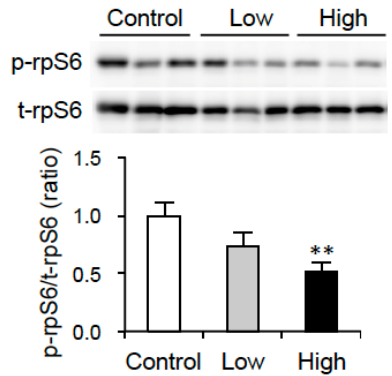

$\mathrm{D}$

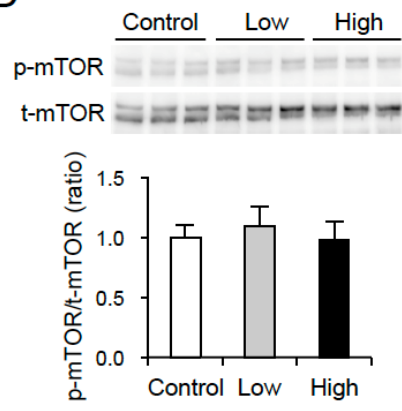

E

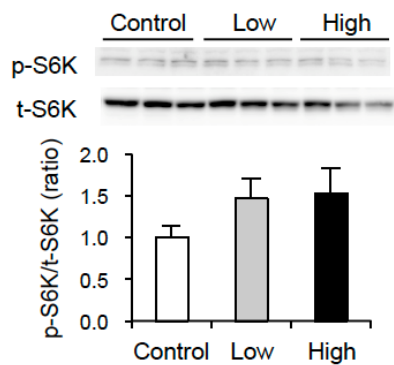

$\mathrm{F}$

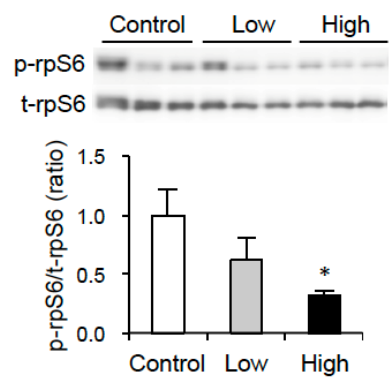

Figure 3. Effect of DT supplementation on mTOR-S6K signaling in WAT and liver of mice. Western blot analyses of mTOR $(\mathbf{A}, \mathbf{D})$, S6K $(\mathbf{B}, \mathbf{E})$, and rpS6 $(\mathbf{C}, \mathbf{F})$ were performed using tissue lysates of EWAT $(\mathbf{A}-\mathbf{C})$ and liver (D-F) from mice in Figure 2. Representative images are shown. Phosphorylation levels of mTOR Ser2448, S6K Thr389, and rpS6 Ser235/236 were normalized to total level of each protein. Data are expressed as mean $\pm \operatorname{SE}(n=5-6) .{ }^{*} p<0.05,{ }^{* *} p<0.01,{ }^{* * *} p<0.001$ vs. Control. ${ }^{\#} p<0.05,{ }^{\# \#} p<0.01$ vs. Low.

\subsection{DT Supplementation Stimulates Expression of Brown Fat-Associated Genes in IBAT of HFD-Fed} Mice at Thermoneutrality

We then examined mRNA expression of brown fat-associated genes in adipose tissues, as the tissue staining indicated that brown adipocyte morphology was well maintained in IBAT of DT-supplemented mice (Figure 2D). In addition to an upregulation of Ucp1 mRNA $(p=0.0628)$, the mRNA levels of cell death-inducing DFFA-like effector A (Cidea), peroxisome proliferator activated receptor- $\gamma$ coactivator 1- $\alpha$ (Ppargc1a), and PR domain containing 16 (Prdm16), a dominant regulator of brown fat cell fate [25], were significantly higher in IBAT in the High group than those in the control group (Figure 4A). 
Moreover, an increase in the Fgf21 mRNA level was found in IBAT in DT-supplemented mice compared to those of the control mice (Figure 4A). Western blot analysis showed significantly higher UCP1 level in IBAT in the High group than the control group (Figure 4D). On the other hand, no significant differences were observed in these mRNA levels in IWAT and EWAT among the three groups (Figure 4B,C). There were no significant differences in the hepatic Fgf21 mRNA and serum FGF21 level among the three groups (Figure 4E,F).

A

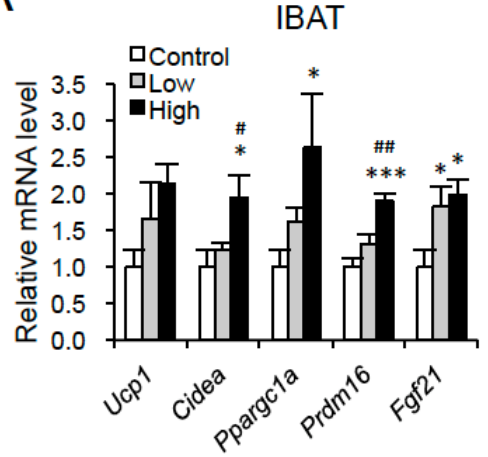

B

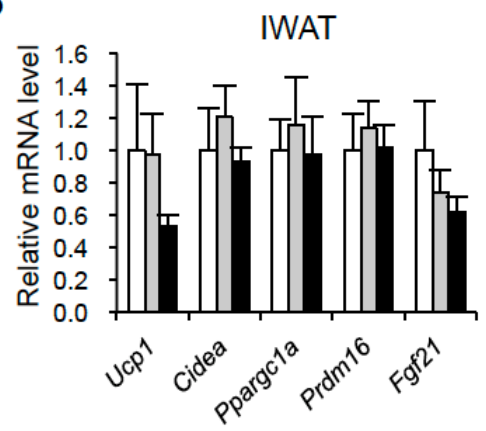

C

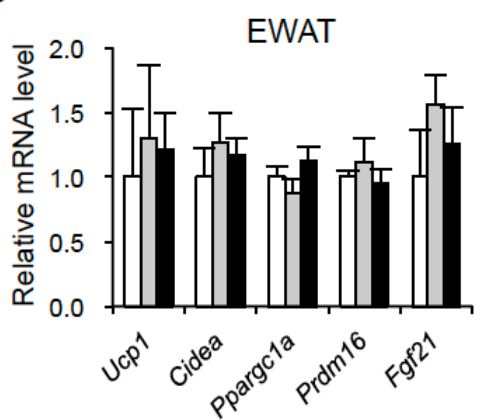

D IBAT
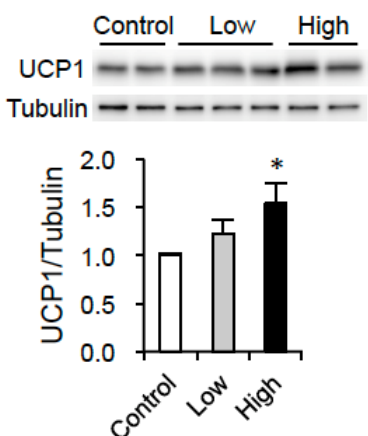

$E$

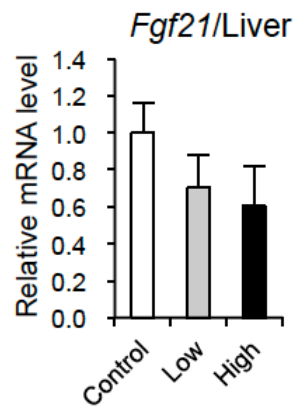

$\mathrm{F}$

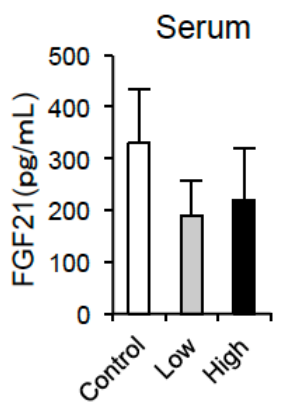

Figure 4. DT supplementation stimulates expression of brown fat-associated genes in IBAT in HFD-fed mice at thermoneutrality. (A-C) mRNA levels of Ucp1, Cidea, Ppargc1a, Prdm16, and Fgf21 in IBAT (A), IWAT (B), and EWAT (C). (D) UCP1 and tubulin levels in IBAT. Representative images are shown. (E) Fgf21 mRNA level in liver. (F) Serum FGF21 level. Data are the means \pm SE $(n=4-6) .{ }^{*} p<0.05$, *** $p<0.001$ vs. Control. ${ }^{\#} p<0.05,{ }^{\# \#} p<0.01$ vs. Low.

\subsection{DT Extract Stimulates Brown Fat-Associated Gene Expression and FGF 21 Production in C3H10T1/2 Adipocytes}

To confirm the stimulatory effect of DT components on brown fat formation, we investigated its effect on the expression of brown fat-associated genes in C3H10T1/2 cells, which is an established in vitro model for brown adipocytes [26]. When adipocytes were treated with DT extract at different concentrations, there was no obvious difference in cell morphology among groups (Figure 5A). However, 
significant upregulation of Ucp1, Cidea, and Prdm16 mRNA expression was found in cells treated with $0.3 \%$ DT extract (Figure 5B,C,E). Gene expressions of Ppargc1a and F $f 21$ were also upregulated when treated with DT extract, which peaked at the concentration of $1.0 \%$ (Figure 5D,F).

A
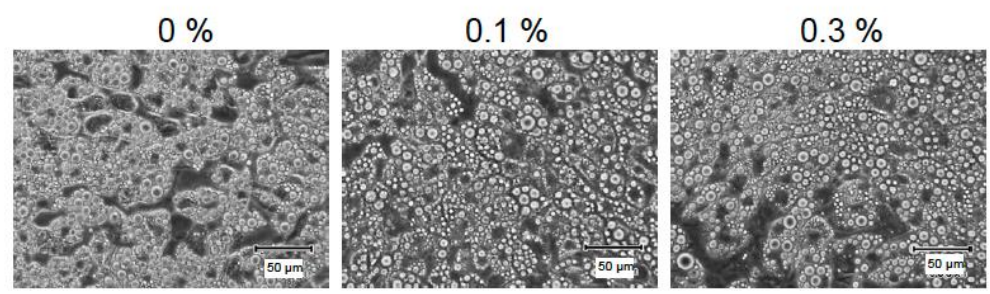

$1 \%$

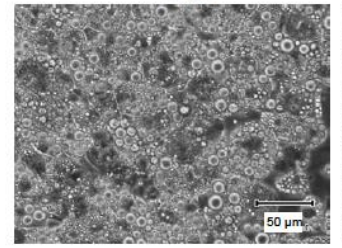

$3 \%$

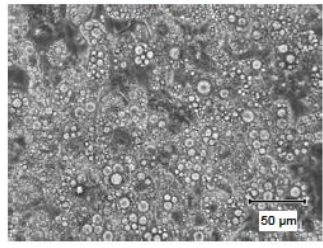

B

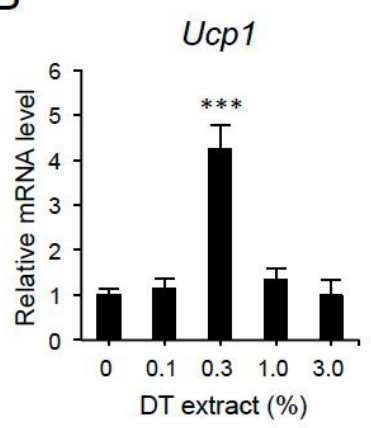

C
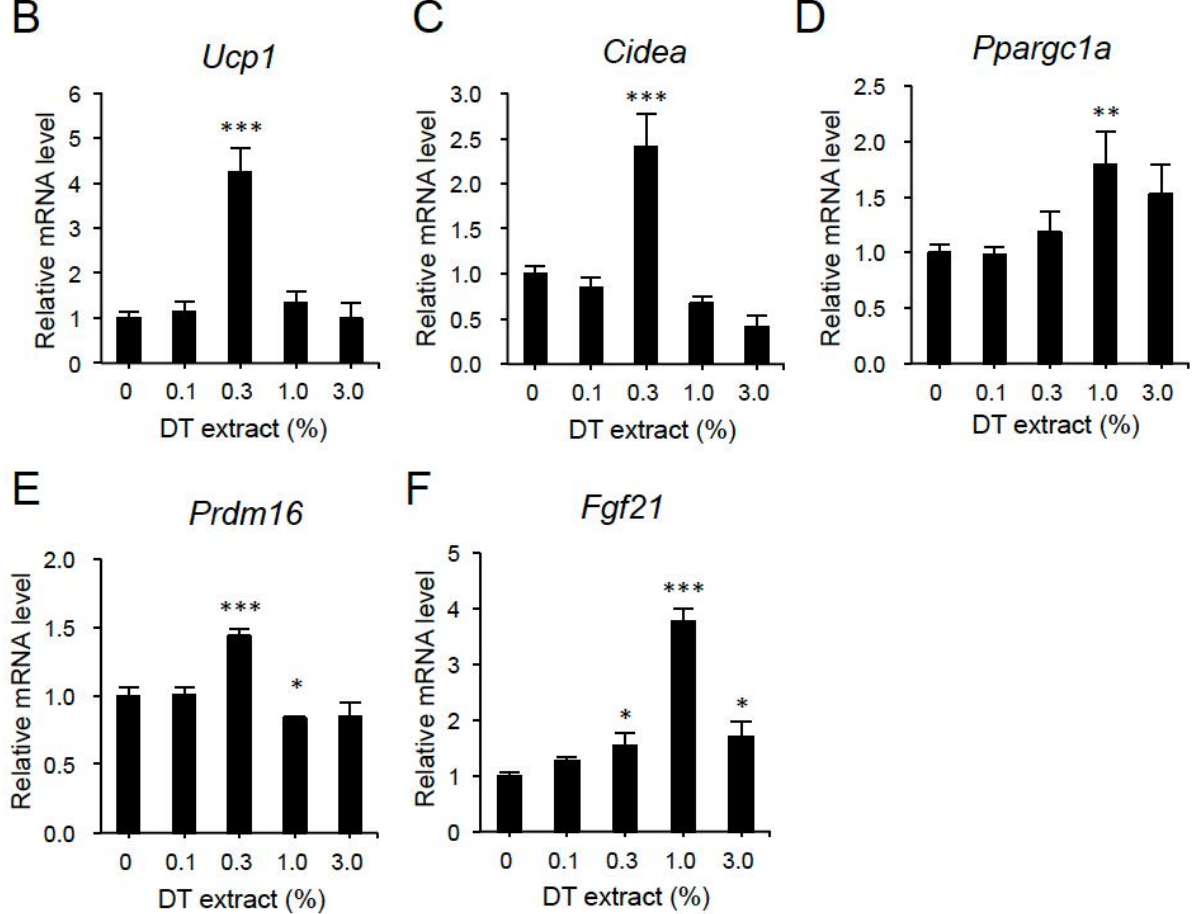

Figure 5. DT extract stimulates expression of brown fat-associated genes in C3H10T1/2 adipocytes. Cells were treated with the indicated concentration of DT extract for $18 \mathrm{~h}$. (A) Cell morphology. (B-F) Dose dependent upregulation of Ucp1 (B), Cidea (C), Ppargc1a (D), Prdm16 (E), and Fgf21 (F) mRNAs. $(n=7$, except $n=4$ for $3 \%)$. Data are the means \pm SE. ${ }^{*} p<0.05,{ }^{* *} p<0.01,{ }^{* * *} p<0.001$ vs. $0 \%$.

In time course experiments carried out using $0.3 \%$ DT extract, the mRNA expressions of Ucp 1 and Fgf21 increased in a time-dependent manner and the mRNA levels at $24 \mathrm{~h}$ were 11.1 and 5.9-fold higher than those at $0 \mathrm{~h}$, respectively (Figure 6A,B). In parallel to $F g f 21$ mRNA expression, a remarkable increase of FGF21 level was detected in the conditioned medium (CM) of adipocytes at $24 \mathrm{~h}$ post treatment with $0.3 \%$ DT extract. However, its level was also elevated without DT stimulation at $24 \mathrm{~h}$ (Figure 6C). We then compared the effect of DT on FGF21 production with that of another green alga, DS. Similar to DT, DS extract increased FGF21 production in a dose-dependent manner in C3H10T1/2 adipocytes, where the FGF21 level in CM was significantly higher (1.8-fold) at the concentration of 
$1.0 \%$ DS than the control (Figure 6D). However, DT induced higher FGF21 production (3.2-fold at $1.0 \%$ ) than that of DS, although its production was significantly inhibited at the concentration of $3.0 \%$ extract in both microalgae (Figure 6D).
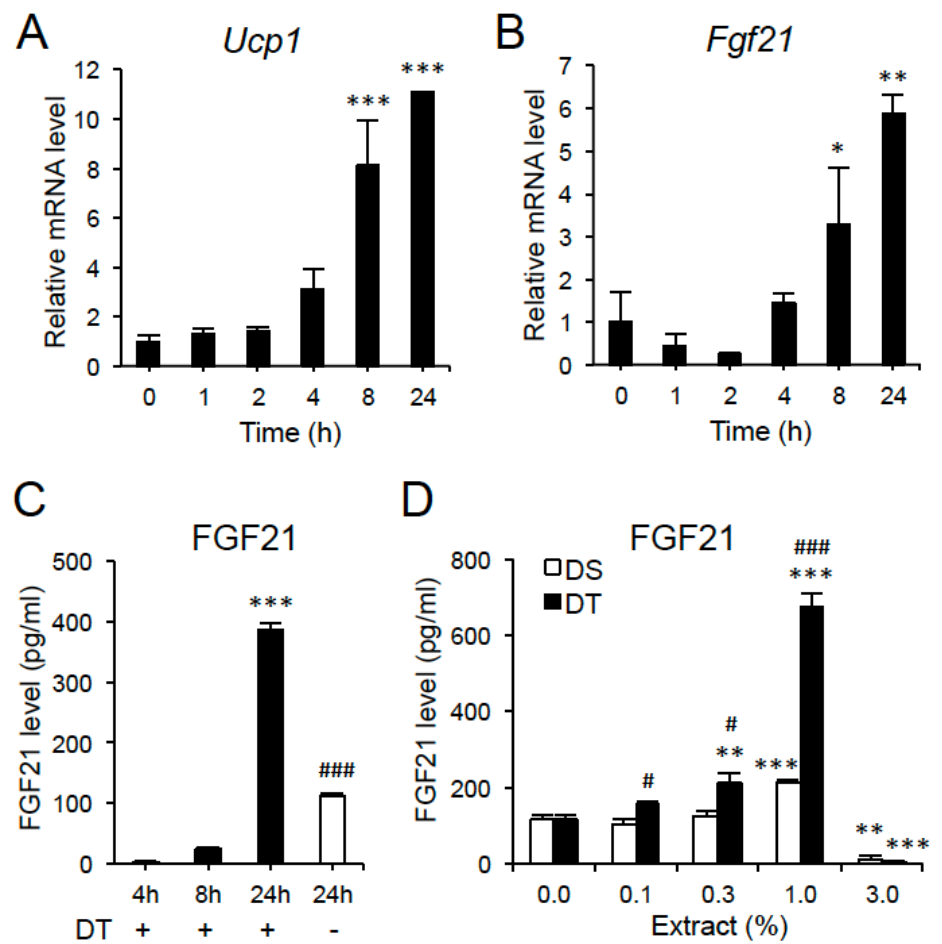

Figure 6. Effects of DT on Ucp1, Fgf21 mRNA expression, and FGF21 production in C3H10T1/2 adipocytes. Cells were stimulated by $0.3 \%$ DT extract for the indicated time (A-C). (A) Ucp1 mRNA level. (B) Fgf21 mRNA level ( $n=2$ for each point, representative of two independent experiments). (C) FGF21 level in the conditioned medium (CM) when treated with DT ( $n=3-5)$. (D) FGF21 level in the CM when treated with DT and DS. Cells were stimulated by the indicated concentration of DT or DS extract for $18 \mathrm{~h}(n=4)$. Data are the means \pm SE. ${ }^{*} p<0.05,{ }^{* *} p<0.01,{ }^{* * *} p<0.001 \mathrm{vs} .0 \mathrm{~h}, 4 \mathrm{~h}$ or $0 \%$ in the same extract. ${ }^{\#} p<0.05,{ }^{\# \#} p<0.001$ vs. DT+ $(24 \mathrm{~h})$ or DS extract at same concentration.

\section{Discussion}

In this study, we showed for the first time, the effectiveness of a green microalga DT as a nutraceutical for the prevention of diet-induced obesity and hepatic lipid accumulation in vivo without any restriction on food intake. The effects of DT are unique and very potent for the following reasons: Firstly, DT supplementation inhibited mTOR-S6K signaling in visceral WAT of mice, which was similar to the results in our previous studies using Evo diet $[17,18]$ and in the study using rapamycin diet [27]. Suppression of mTOR signaling is thought to mediate major beneficial effects of caloric restriction including suppression of obesity, type 2 diabetes, cancer, and neurodegeneration [28,29]. S6K phosphorylated by mTOR promotes mRNA translation by phosphorylating multiple proteins such as eukaryotic translation initiation factor $4 \mathrm{~B}$ and $\mathrm{rpS6}$, which is involved in the control of mammalian cell size [30]. Therefore, the significant reduction in rpS6 phosphorylation in WAT and liver by DT supplementation might contribute to suppression of cell growth, consequently contributing to the prevention of lipid accumulation in these tissues of mice.

Secondly, DT supplementation notably inhibited BAT whitening (i.e., attenuation of brown fat) in mice at thermoneutrality. This finding was most intriguing because UCP1 expression and BAT thermogenic function are usually attenuated to a great extent, accelerating susceptibility to obesity in the thermoneutral condition [31,32]. The stimulatory effect of DT for brown fat formation was confirmed in a brown adipocyte culture model, in which the microalga extract stimulated brown fat-selective genes 
such as Ucp1 and $F g f 21$ in dose-dependent and time-dependent manners. Although we failed to detect an increase of FGF21 level in serum of DT supplemented mice, DT extract significantly increased the secretion of FGF21 in C3H10 adipocyte culture. Interestingly, the effect of DT on FGF21 production was greater than that of DS, which belongs to the same strain. This secreted FGF21 may stimulate brown fat formation, including UCP1 expression in an autocrine/paracrine fashion as reported previously [8]. Véniant et al. reported that FGF21 administration potently prevents whitening and induces UCP1 expression in BAT even at thermoneutrality [33]. FGF21 administration also improves glucose clearance, which requires UCP1 thermogenesis in mice [34]. Although the role of FGF21 appears to be complex because FGF21 performs diverse metabolic functions in multiple target organs [35], the metabolic benefits provided by FGF21 may have pharmacological significance in improving obesity and insulin resistance [36]. Thus, our results suggest that DT administration may stimulate FGF21 induction in BAT and thereby bolster the FGF21 autocrine/paracrine mechanism-mediated differentiation of brown preadipocytes.

Regarding the compounds capable of promoting brown fat formation, carotenoid can be a candidate responsible for the effects of DT. Serra et al. reported that $10 \mu \mathrm{M}$ concentrations of carotenoids, including $\beta$-carotene and lutein, upregulated UCP1 expression in brown adipocytes [37]. $\beta$-carotene is enzymatically converted to vitamin A (retinol), and this lipophilic vitamin and its derivatives play an important role in adipocyte biology, due to which this carotenoid has attracted attention in the field of obesity research [38]. Essentially, retinoic acid upregulates UCP1 expression in adipocytes differentiated from mouse embryonic fibroblasts [39], despite blocking adipogenesis in 3T3-L1 preadipocyte, a white adipocyte culture model [40]. However, the concentrations of $\beta$-carotene and lutein in DT extract were 13 and $211 \mu \mathrm{M}$, respectively, which reduces to 0.039 and $0.633 \mu \mathrm{M}$ in $0.3 \%$ extract. In addition, their concentrations were significantly higher in DS extracts ( $\beta$-carotene: $3.35 \mathrm{mM}$, lutein: $580 \mu \mathrm{M}$ ). Therefore, it is plausible that the combination of compounds including carotenoids in DT promotes brown fat formation.

Our findings strongly suggest that DT holds nutraceutical potential for the prevention of obesity and metabolic diseases by regulating adipose tissue functions including stimulation of brown fat formation via FGF21 production. Considering the crucial role of brown fat in energy metabolism and the causal relation between the decline in brown fat activity and obesity progression, DT may serve as a functional food for human health. Further studies may reveal unknown effective compounds involved in brown fat formation and FGF21 production in future study.

Author Contributions: H.Y. conceptualized and designed the experiments. S.S. and Y.Y. (Yuji Yamaguchi) prepared microalgae powder and extracts and analyzed evodiamine and carotenoids, supervised by H.T., Y.Y. (Yukari Yamashita), Y.E., A.G., and H.Y. performed animal experiments. T.T. analyzed H\&E samples. Y.Y. (Yukari Yamashita) and H.Y. performed cell culture experiments and analyzed data. H.Y. wrote the manuscript. All authors have read and agreed to the published version of the manuscript.

Funding: This research was funded in part by a grant from the MEXT-Supported program for Strategic Research Foundation at private universities (S1201007).

Acknowledgments: We thank Y. Ohmi, S. Tsunoda, and K. Hori for technical assistance.

Conflicts of Interest: The authors declare no conflict of interest regarding the publication of this article.

\section{References}

1. Kolonin, M.G.; Saha, P.K.; Chan, L.; Pasqualini, R.; Arap, W. Reversal of obesity by targeted ablation of adipose tissue. Nat. Med. 2004, 625-632. [CrossRef]

2. Enerbäck, S.; Jacobsson, A.; Simpson, E.M.; Guerra, C.; Yamashita, H.; Harper, M.E.; Kozak, L.P. Mice lacking mitochondrial uncoupling protein are cold-sensitive but not obese. Nature 1997, 387, 90-94. [CrossRef]

3. Cannon, B.; Nedergaard, J. Brow adipose tissue: Function and physiological significance. Physiol. Rev. 2004, 84, 277-359. [CrossRef] 
4. Kontani, Y.; Wang, Y.; Kimura, K.; Inokuma, K.I.; Saito, M.; Suzuki-Miura, T.; Wang, Z.; Sato, Y.; Mori, N.; Yamashita, H. UCP1 deficiency increases susceptibility to diet-induced obesity with age. Aging Cell 2005, 4, 147-155. [CrossRef]

5. Cypess, A.M.; Lehman, S.; Williams, G.; Tal, I.; Rodman, D.; Goldfine, A.B.; Kuo, F.C.; Palmer, E.L.; Tseng, Y.H.; Doria, A.; et al. Identification and importance of brown adipose tissue in adult humans. N. Engl. J. Med. 2009, 360, 1509-1517. [CrossRef]

6. Tseng, Y.H.; Cypess, A.M.; Kahn, R. Cellular bioenergetics as a target for obesity therapy. Nat. Rev. Drug Discov. 2010, 9, 465-481. [CrossRef] [PubMed]

7. Harms, M.; Seale, P. Brown and beige fat: Development, function and therapeutic potential. Nat. Med. 2013, 19, 1252-1263. [CrossRef]

8. Fisher, F.M.; Kleiner, S.; Douris, N.; Fox, E.C.; Mepani, R.J.; Verdeguer, F.; Wu, J.; Kharitonenkov, A.; Flier, J.S.; Maratos-Flier, E.; et al. FGF21 regulates PGC-1 $\alpha$ and browning of white adipose tissues in adaptive thermogenesis. Gene Dev. 2012, 26, 271-281. [CrossRef]

9. Alsenani, F.; Ahmed, F.; Schenk, P.M. Nutraceuticals from microalgae. In Nutraceuticals and Functional Foods in Human Health and Disease Prevention, 1st ed.; Bagchi, D., Preuss, H.G., Swaroop, A., Eds.; CRC Press: Boca Raton, FL, USA, 2015; pp. 673-684.

10. Hamed, I.; Özogul, F.; Özoğul, Y.; Regenstein, J.M. Marine bioactive compounds and their health benefits: A Review. Compr. Rev. Food. Sci. Food Saf. 2015, 14, 446-465. [CrossRef]

11. Kumar, S.A.; Magnusson, M.; Ward, L.C.; Paul, N.A.; Brown, L. A green algae mixture of Scenedesmus and Schroederiella attenuates obesity-linked metabolic syndrome in rats. Nutrients 2015, 7, 2771-2787. [CrossRef]

12. Gille, A.; Stojnic, B.; Derwenskus, F.; Trautmann, A.; Schmid-Staiger, U.; Posten, C.; Briviba, K.; Palou, A.; Bonet, M.L.; Ribot, J. A lipophilic fucoxanthin-rich Phaeodactylum tricornutum extract ameliorates effects of diet-induced obesity in C57BL/6J Mice. Nutrients 2019, 11, 796. [CrossRef]

13. Bauer, R.; Xiao, P.G. Fructus Evodiae Wuzhuyu. In Chromatographic Fingerprint Analysis of Herbal Medicines; Wagner, H., Bauer, R., Melchart, D., Xiao, P.G., Staudinger, A., Eds.; Springer Nature: Vienna, Austria, 2011; pp. 391-401.

14. Chiou, W.F.; Chou, C.J.; Shum, A.Y.; Chen, C.F. The vasorelaxant effect of evodiamine in rat isolated mesenteric arteries: Mode of action. Eur. J. Pharmacol. 1992, 215, 277-283. [CrossRef]

15. Takada, Y.; Kobayashi, Y.; Aggarwal, B.B. Evodiamine abolishes constitutive and inducible NF-kappaB activation by inhibiting IkappaBalpha kinase activation, thereby suppressing NF-kappaB-regulated antiapoptotic and metastatic gene expression, up-regulating apoptosis, and inhibiting invasion. J. Biol. Chem. 2005, 280, 17203-17212. [CrossRef]

16. Wang, T.; Wang, Y.; Kontani, Y.; Kobayashi, Y.; Sato, Y.; Mori, N.; Yamashita, H. Evodiamine improves diet-induced obesity in a uncoupling protein-1-independent manner: Involvement of antiadipogenic mechanism and extracellularly regulated kinase/mitogen-activated protein kinase signaling. Endocrinology 2008, 149, 358-366. [CrossRef] [PubMed]

17. Wang, T.; Kusudo, T.; Takeuchi, T.; Yamashita, Y.; Kontani, Y.; Okamatsu, Y.; Saito, M.; Mori, N.; Yamashita, H. Evodiamine inhibits insulin-stimulated mTOR-S6K activation and IRS1 serine phosphorylation in adipocytes and improves glucose tolerance in obese/diabetic mice. PLoS ONE 2013, 8, e83264. [CrossRef]

18. Yamashita, H.; Kusudo, T.; Takeuchi, T.; Qiao, S.; Tsutsumiuchi, K.; Wang, T.; Wang, Y. Dietary supplementation with evodiamine prevents obesity and improves insulin resistance in ageing mice. J. Funct. Foods 2015, 19, 320-329. [CrossRef]

19. Sakaki, S.; Tsutsumiuchi, K.; Yamaguchi, Y.; Yamashita, H.; Takenaka, H. Identification of evodiamine in the microalga Dunaliella tertiolecta (Chlorophyceae). New Food Indust. 2018, 60, 13-18.

20. Berner, T.; Dubinsky, Z.; Wyman, K.; Falkowski, P.G. Photo-adaptation and the "package" effect in Dunaliella tertiolecta (Chloropyneae). J. Phycol. 1989, 25, 70-78. [CrossRef]

21. Villar, R.; Laguna, M.R.; Calleja, J.M.; Cadavid, I. Effects of Phaedactylum tricornutum and Dunaliella tertiolecta extracts on the central nervous system. Planta Med. 1992, 58, 405-409. [CrossRef]

22. Fabregas, J.; Herrero, C.; Gamallo, Y.; Otero, A.; Paz, M.H.; Vecino, E. Decrease of plasma cholesterol with the marine microalga Dunaliella tertiolecta in hyper cholesterolemic rats. J. Gen. Appl. Microbiol. 1994, 40, 533-540. [CrossRef] 
23. Villar, R.; Laguna, R.; Martinez, D.; Nunez, L.; Jimenez, C. Anti-aggregant effects on human platelets of the crude aqueous extract and polar fractions of the microalga Dunlaiella tertiolecta. Phytother. Res. 1997, 11, 70-72. [CrossRef]

24. Kusudo, T.; Wang, Z.; Mizuno, A.; Suzuki, M.; Yamashita, H. TRPV4 deficiency increases skeletal muscle metabolic capacity and resistance against diet-induced obesity. J. Appl. Physiol. 2012, 112, 1223-1232. [CrossRef]

25. Seale, P.; Bjork, B.; Yang, W.; Kajimura, S.; Chin, S.; Kuang, S.; Scimè, A.; Devarakonda, S.; Conroe, H.M.; Erdjument-Bromage, H.; et al. PRDM16 controls a brown fat/skeletal muscle switch. Nature 2008, 454, 961-967. [CrossRef] [PubMed]

26. Brunmeir, R.; Wu, J.; Peng, X.; Kim, S.Y.; Julien, S.G.; Zhang, Q.; Xie, W.; Xu, F. Comparative transcriptomic and epigenomic analyses reveal new regulators of murine brown adipogenesis. PLoS Genet. 2016, 12, e1006474. [CrossRef] [PubMed]

27. Harrison, D.E.; Strong, R.; Sharp, Z.D.; Nelson, J.F.; Astle, C.M.; Flurkey, K.; Nadon, N.L.; Wilkinson, J.E.; Frenkel, K.; Carter, C.S.; et al. Rapamycin fed late in life extends lifespan in genetically heterogeneous mice. Nature 2009, 460, 392-395. [CrossRef]

28. Kenyon, C.J. The genetics of ageing. Nature 2010, 464, 504-512. [CrossRef] [PubMed]

29. Zoncu, R.; Efeyan, A.; Sabatini, D.M. mTOR: From growth signal integration to cancer, diabetes and ageing. Nat. Rev. Mol. Cell Biol. 2011, 12, 21-35. [CrossRef]

30. Fingar, D.C.; Salama, S.; Tsou, C.; Harlow, E.; Blenis, J. Mammalian cell size is controlled by mTOR and its downstream targets S6K1 and 4EBP1/eIF4E. Genes Dev. 2002, 16, 1472-1487. [CrossRef]

31. Liu, X.; Rossmeisl, M.; McClaine, J.; Leslie, P.K. Paradoxical resistance to diet-induced obesity in UCP1-deficient mice. J. Clin. Invest. 2003, 111, 399-407. [CrossRef]

32. Kataoka, N.; Takeuchi, T.; Kusudo, T.; Li, Y.; Endo, Y.; Yamashita, H. Lack of UCP1 stimulates fatty liver but mediates UCP1-independent action of beige fat to improve hyperlipidemia in Apoe knockout mice. Biochim. Biophys. Acta Mol. Basis Dis. 2020, 1866, 165762. [CrossRef]

33. Véniant, M.M.; Sivits, G.; Helmering, J.; Komorowski, R.; Lee, J.; Fan, W.; Moyer, C.; Lloyd, D.J. Pharmacologic effects of FGF21 are independent of the "Browning" of white adipose tissue. Cell Metab. 2015, 21, 731-738. [CrossRef] [PubMed]

34. Kwon, M.M.; O'Dwyer, S.M.; Baker, R.K.; Covey, S.D.; Kieffer, T.J. FGF21-mediated improvements in glucose clearance require uncoupling protein 1. Cell Rep. 2015, 13, 1521-1527. [CrossRef]

35. Fisher, F.M.; Maratos-Flier, E. Understanding the physiology of FGF21. Annu. Rev. Physiol. 2016, 78, $223-241$. [CrossRef]

36. Ni, B.; Farrar, J.S.; Vaitkus, J.A.; Celi, F.S. Metabolic Effects of FGF-21: Thermoregulation and Beyond. Front. Endocrinol. Lausanne 2015, 6, 148. [CrossRef]

37. Serra, F.; Bonet, M.L.; Puigserver, P.; Oliver, J.; Palou, A. Stimulation of uncoupling protein 1 expression in brown adipocytes by naturally occurring carotenoids. Int. J. Obes. Relat. Metab. Disord. 1999, 23, 650-655. [CrossRef]

38. Coronel, J.; Pinos, I.; Amengual, J. $\beta$-carotene in Obesity Research: Technical Considerations and Current Status of the Field. Nutrients 2019, 11, 842. [CrossRef] [PubMed]

39. Mercader, J.; Palou, A.; Bonet, M.L. Induction of uncoupling protein-1 in mouse embryonic fibroblast-derived adipocytes by retinoic acid. Obes. Silver Spring 2010, 18, 655-662. [CrossRef] [PubMed]

40. Schwarz, E.J.; Reginato, M.J.; Shao, D.; Krakow, S.L.; Lazar, M.A. Retinoic acid blocks adipogenesis by inhibiting C/EBPbeta-mediated transcription. Mol. Cell Biol. 1997, 17, 1552-1561. [CrossRef] [PubMed]

(C) 2020 by the authors. Licensee MDPI, Basel, Switzerland. This article is an open access article distributed under the terms and conditions of the Creative Commons Attribution (CC BY) license (http://creativecommons.org/licenses/by/4.0/). 